\title{
Development of a Multisite Distribution Rotation for Pharmacy Practice Residents
}

\author{
Anita Lo and Edward C Dillon
}

\section{INTRODUCTION}

$\mathrm{T}$ he distribution rotation is one of the compulsory rotations in the Canadian pharmacy practice residency program. This rotation aligns with the 2010 Accreditation Standards of the Canadian Hospital Pharmacy Residency Board (CHPRB), which require pharmacy practice residents to demonstrate knowledge of medication-use systems. ${ }^{1}$ All residency training programs across Canada incorporate a distribution rotation, which runs from 2 to 8 weeks. $^{2}$ The rotation introduces pharmacy practice residents to dispensary functions, medication distribution systems, IV admixtures, and basic computer applications.

As regionalization evolves and becomes a widely accepted health care structure in many provinces in Canada, ${ }^{3-5}$ there will be more instances of pharmacy sites with different drug distribution systems being amalgamated into one department under one health authority. If a pharmacy practice resident is to meet the CHPRB standards and be effectively integrated into the organization's staff complement at any site once the residency program ends, he or she must be introduced to as many of the drug distribution systems and sites as possible during the distribution rotation. In recognition of this reality, a multisite distribution rotation was designed by the Fraser Health Authority in British Columbia, where 12 pharmacy sites have been merged to create a regional, one-department module.

\section{DEVELOPMENT OF DISTRIBUTION ROTATION}

To ensure that pharmacy practice residents were introduced to the various drug distribution systems and practice settings within the Fraser Health Authority, potential preceptors from multiple sites were encouraged to act as rotation facilitators. A schedule for a 4-week rotation was designed to ensure exposure to all of the distribution systems used within the department. The schedule included exposure to advanced technology in packaging, integration of centralized purchasing, inventory control, and delivery of patient-specific medications. The distribution rotation is the first compulsory rotation for all pharmacy practice residents in Fraser Health; the residency also includes 10 additional 4-week rotations (of which 3 are compulsory), two 2-week rotations, and 2 weeks of didactic instruction.

Because some hospital sites had identical drug distribution systems, it was not necessary for residents to visit every site, and only 8 of the 12 sites were included in the final rotation schedule. Despite certain differences from one site to another, goals and objectives were common across all of the sites. The goals met the CHPRB Accreditation Standards, and the objectives, quoted directly from the standards document, were formulated in a measurable format (see Appendix 1).

One of the sites that pharmacy practice residents were required to visit as part of the distribution rotation was the Pharmacy Drug Distribution Centre. This centre was created as part of the Fraser Health Authority Pharmacy's plan, adopted in February 2004, to reduce adverse drug events and thus improve medication safety. The centre incorporated an automated unit-dose drug distribution system, as well as an IV admixture-based system for acute and residential care sites. Purchasing and inventory control were also centralized within the Pharmacy Drug Distribution Centre. The centre was connected through an electronic network to all of the Fraser Health Authority sites and hosted state-of-the-art technology. The residents' objective related to the Pharmacy Drug Distribution Centre was to learn how automation can improve medication safety, enhance the ergonomic aspects of drug distribution, and add efficiencies.

In addition to the specific objectives for the Pharmacy Drug Distribution Centre, the following sites had their own specific objectives for the residents (beyond the objectives common to all sites):

- Langley Memorial Hospital, a site with hand-held scanners for bar-coded ward stock (a system for the safe 
and effective management of pharmaceutical inventory): learn how inventory is charged and ordered with the scanners

- Royal Columbian Hospital, a site with total parenteral nutrition pumps: learn the advantages and limitations of the pumps and be able to describe how the pump operates

- Eagle Ridge Hospital, a site with a full manual unit-dose system: learn how to run unit-dose refill lists, the significance of cutoff times and exchange bins, and how a system of this type reduces errors

- Burnaby Hospital, a site with an oncology admixture service: learn how to mix oncology drugs and how to safely handle hazardous medications

- Abbotsford Regional Hospital, a site with an automated dispensing cabinet: learn how information from pharmacy profiles flows to the cabinet and the advantages of controlled narcotic ward stock

These objectives went beyond what is required by the accreditation standard that reads "The department shall provide a safe and effective drug distribution system for all medications used within the organization in a manner consistent with the patient population(s) being served" (page 7).

In 2009/2010, the second year of the fully integrated multisite distribution rotation, the Fraser Health Authority accepted 10 residents. Two of these were former staff members (one a former technician and the other a former pharmacist), who were exempted from the distribution rotation because of their familiarity with the systems. The remaining 8 residents followed the newly created schedule for the rotation. Each resident was assigned to a "home site", where he or she received basic orientation (common to all residents) relating to regional policies and procedures, the telephone and on-call systems, electronic night cupboards, narcotic cupboards, fundamental order-entry processes, etc. A resident's assigned home site determined his or her need to visit other sites, as outlined in the schedule.

Input was solicited from the preceptors at the sites mentioned above to determine the time required for a resident to learn the particular skills at each site. With the exception of the Pharmacy Drug Distribution Centre and the Abbotsford Regional Hospital, where 2-day visits were requested, the preceptors at each of the above-mentioned sites requested a 1-day visit for each resident. At the end of the 1- or 2-day visit to a particular site, the residents were expected to understand, and to have had some hands-on experience with, the equipment or system introduced at that site. To ensure that the overall objectives of the distribution rotation were satisfied, the site preceptors were asked to create complementary sets of questions and answers. For example, after visiting the site that introduced the automated dispensing machine (Abbotsford Regional Hospital), the resident would be asked to describe how to perform critical and emergent overrides. The questions for a particular site were posed at the end of the 1- or 2-day visit, and residents had to reach an acceptable predetermined performance level in answering these questions to demonstrate their compliance with the objectives of the rotation. A resident's performance level for visits to locations other than the home site was then reported to the preceptor at the primary site by e-mail. The resident's competency for the entire distribution rotation was measured by means of a comprehensive oral exam (based on the specific objectives) at the end of the rotation.

Preceptors at all sites were informed at the outset that their sites might or might not be included on the final schedule. Before the schedule was set up, all potential preceptors were asked to indicate the best day of the week for residents to visit, the number of days required for the optimal experience, and the number of residents that could be accommodated. Draft schedules were sent to the preceptors for confirmation, and the final schedule was sent to the residency coordinator for assignment of residents to the various home sites. No input on scheduling was solicited from the residents.

\section{EVALUATION}

To measure satisfaction with the design of the distribution rotation, a 10-question survey was distributed (via Survey Monkey) to the 8 residents and the 10 site preceptors at the end of the rotation. The response rate was $100 \%$ for both residents and preceptors.

Both residents and preceptors felt that after the rotation, the residents were equipped with knowledge of the various distribution systems, including the unit-dose system, the traditional dosing system, and the automated dispensing system. The residents also felt confident that they could work at any Fraser Health Authority site (Figure 1). This confidence would not imply that a resident could start working at any site without a refresher orientation, but it was anticipated that the visits to multiple sites during the residency would decrease the amount of time required to familiarize a newly hired residencytrained pharmacist to any of the 12 sites. Both residents and preceptors agreed that learning about the various distribution systems was valuable and that the question-and-answer format used to measure learning was helpful. The majority of residents (5 of 8 [62\%]) and preceptors (7 of $10[70 \%])$ preferred that residents not stay at any one site for the duration of the rotation. Although more than half of the preceptors ( 4 of 7 [57\%]) indicated that they would not change the current schedule, a similar proportion of the residents (5 of 8 [62\%]) indicated that they would like to see some changes. Three (38\%) of the 8 residents felt that the visit to the Langley Memorial Hospital, where the hand-held scanning technology was introduced, was unnecessary. Overall, both residents and preceptors indicated a willingness to recommend the 


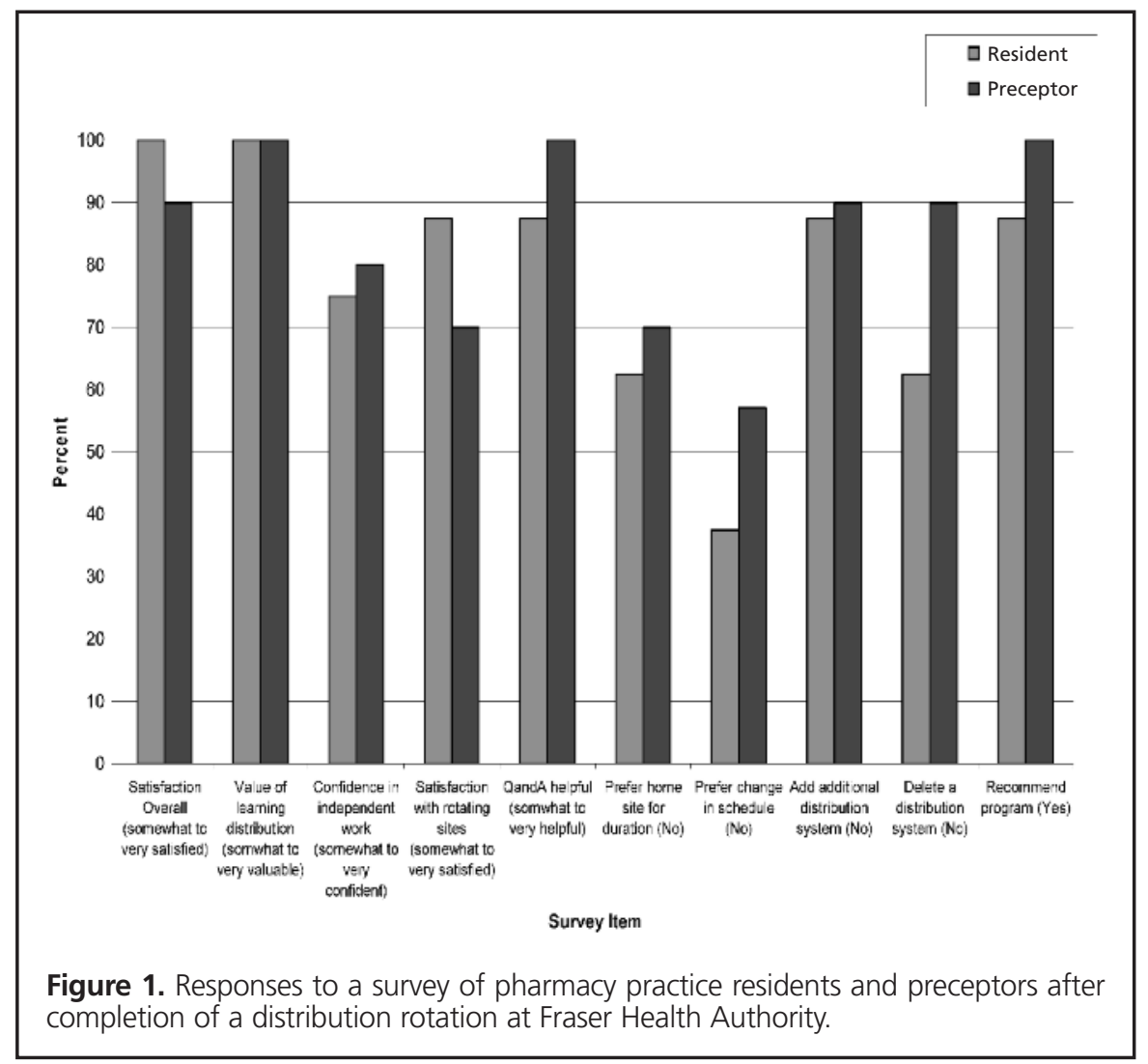

distribution rotation of the Fraser Health Authority to all future residents.

\section{DISCUSSION}

Although pharmacy practice residency programs devote more time to clinical activities than to other aspects of pharmacy practice, exposure to various drug distribution systems is a requirement for all Canadian programs. We tried to ensure that our rotation enabled residents to acquire knowledge of different medication systems, as required by the CHPRB. We also tried to prepare our residents for integration into the staff complement at any Fraser Health Authority pharmacy site upon completion of the residency program. Acquisition of desired knowledge was verified at the time of exposure through assessment questions. Success in meeting the objectives of the rotation was assessed through a survey of residents and preceptors, the results of which indicated achievement of our goal. Given the challenges of developing high-quality experiences for a growing number of residents and given that regionalization and consolidation are continuing within the pharmacy profession, the Fraser Health Authority multisite distribution rotation may be suitable as a prototype for other regions. The set-up of the first multisite schedule was somewhat cumbersome, but subsequent schedules have been easier to coordinate.
As with the development of any rotation or schedule, the shortcomings of our methodology and findings should be considered by others planning to implement a similar rotation. First, administrators assumed that a visit to the state-of-the art Pharmacy Drug Distribution Centre would be favourably received and that an introduction to the hand-held scanners would be appealing. However, residents had the opposite reaction to these aspects of the rotation. Second, as the number of sites to which a resident was exposed increased over the 4week distribution rotation, more time had to be devoted to site visits, at the expense of learning opportunities at the home site. Before implementation of the multisite distribution rotation, when the entire residency was conducted at a single site, there was more time to learn IV admixture procedures, parenteral nutrition methods, and procedures for medical emergencies (such as borrowing supplies of narcotics from local drugstores on an urgent basis, responding to "code" situations, and handling on-call emergent or urgent questions) at the home site. These experiences were deemed important to residents. ${ }^{6}$ Third, time was lost and costs (such as parking and mileage) were incurred by residents' travel to different sites. Fourth, evaluation of the success of the rotation was based solely on a 10-question survey that was specific to the Fraser Health Authority. Finally, it was not within the scope of this article to address the "retention of understanding" associated with such a 
rotation. The comprehensive examination at the end of the pharmacy practice residency focuses on direct patient care responsibilities, whereas competency related to drug distribution is assessed at the end of the distribution rotation. Notably, the CHPRB Accreditation Standards do not require an assessment of distribution competency at the end of the program.

Most of the unique distribution equipment described above is handled and operated by pharmacy technicians at the various sites. As technicians' scope of practice advances, the need for pharmacists to work in distribution will probably become less important, although an understanding of the various distribution systems will still be needed.

\section{References}

1. Canadian Hospital Pharmacy Residency Board. Accreditation standards January 2010. Ottawa (ON): Canadian Society of Hospital Pharmacists; 2009 [cited 2010 May]. Available from: www.cshp.ca/programs/ residencyTraining/CHPRB_Standards_2010_-_FINAL.pdf

2. Residency training: CHPRB accredited programs. Ottawa (ON): Canadian Society of Hospital Pharmacists; 2010 [cited 2010 May]. Available from: www.cshp.ca/programs/residencyTraining/accreditedPrograms/ index_e.asp

3. Davis J. Let regionalization continue to evolve. Healthc Pap 2004;5(1): 50-54, discussion 96-99.

4. Lewis S, Kouri D. Regionalization: making sense of the Canadian experience, Healthc Pap 2004:5(1):12-31.
5. Church J, Barker P. Regionalization of health services in Canada: a critical perspective. Int J Health Serv 1998;28(3):467-486.

6. Garris K, Wellein MG, Wessell A, Ragucci D, Blair MM. First-year residency candidates' experience in various areas of pharmacy practice. $A m$ J Pharm Educ 2008;72(1):Article 06.

Anita Lo, BSc(Pharm), PharmD, is Pharmacy Manager/Clinical Practice Leader at Eagle Ridge Hospital, Port Moody, British Columbia.

Edward C Dillon BA, BSC, BSC(Pharm), ACPR, PharmD, is Student Education and Residency Coordinator, Department of Pharmacy, Fraser Health Authority, New Westminster, British Columbia, and Clinical Professor, Faculty of Pharmaceutical Sciences, The University of British Columbia, Vancouver, British Columbia.

\section{Address correspondence to:}

Dr Anita Lo

Eagle Ridge Hospital

475 Guildford Way

Port Moody BC V3H 3W9

e-mail: anita.lo@fraserhealth.ca

\section{Acknowledgements}

The authors wish to acknowledge Michael Wasdell for his help with the collection of data and Stephanie Lo for proofreading the article in advance of submission.

Appendix 1. Objectives of the distribution rotation within the pharmacy practice residency program of the Fraser Health Authority (FH). PCA = patient-controlled analgesia, TPN = total parenteral nutrition. (c) 2008 Fraser Health Authority Pharmacy Practice Residency Program. Reproduced by permission.

\section{OBJECTIVES}

At the end of the rotation, the resident will be able to:

1. Demonstrate the ability to locate and appropriately (as defined by the preceptor) interpret source documentation outlining the legal requirements and professional standards that pertain to the distribution and control of drugs in health care organizations at least 3 times during the rotation.

2. Demonstrate the ability to find and appropriately (as defined by the preceptor) interpret 3 assigned FH policy and/or procedure documents relating to drug distribution and administration.

3. Name 3 different types of drug distribution systems in place within FH. Describe 3 key principles of each system and list 3 advantages and 3 disadvantages of each of these systems.

4. Describe 3 roles of the drug distribution process as a component of the provision of pharmaceutical care.

5. Identify 3 potential drug-related problems that could occur within FH's distribution system and identify 3 ways to prevent their occurrence.

6 . Describe 3 roles of pharmacy technicians and 3 roles of 2 other support personnel in the functioning of the drug distribution system.
7. Identify 10 patient-specific, real or potential, drug-related problems through the use of medication profiles and document the appropriate action taken to resolve these problems.

8. State 3 benefits and 3 limitations of using a profile for pharmacotherapy monitoring and drug distribution.

9. Demonstrate at least one process of medication incident reporting and analysis.

10. Demonstrate 3 basic principles of aseptic technique under direct observation.

11. Describe 3 advantages and 3 limitations of a pharmacybased intravenous admixture service.

12. Describe 3 specialized equipment used in an intravenous admixture service, including TPN, and describe 3 techniques and 2 aspects of the technology that may be used to increase efficiency and productivity of the service.

13. Describe all precautions that are unique to cytotoxic drug admixture and delivery.

14. Describe 3 other types of parenteral drug delivery systems and programs (e.g., PCA, home IV therapy, etc.). 